\title{
Are "U-shape" age profiles a universal feature in spiral galaxies?
}

\author{
Estrella Florido $o^{1,2}$, Tomás Ruiz-Lara ${ }^{1,2}$, Gareth Few ${ }^{3}$, Brad Gibson ${ }^{4}$, \\ Isabel Pérez ${ }^{1,2}$, Ivan Minchev ${ }^{5}$ and Patricia Sánchez-Blázquez ${ }^{6}$ \\ ${ }^{1}$ Dpto. de Física Teórica y del Cosmos, Universidad de Granada, Campus de Fuentenueva, \\ E-18071 Granada, Spain. email: estrella@ugr.es, ruizlara@ugr.es, isa@ugr.es \\ ${ }^{2}$ Instituto Carlos I de Física Teórica y computacional, Universidad de Granada, E-18071 \\ ${ }^{3}$ School of Physics, University of Exeter, Stocker Road, Exeter EX4 4QL, UK \\ ${ }^{4}$ E.A. Milne Centre for Astrophysics, Department of Physics \& Mathematics, University of \\ Hull, Hull, HU6 7RX, UK \\ ${ }^{5}$ Leibniz-Institut für Astrophysik Potsdam, An der Sternwarte 16, 14482 Potsdam, Germany \\ ${ }^{6}$ Dpto. de Física Teórica, Universidad Autónoma de Madrid, E-28049 Cantoblanco, Spain
}

In this work we study the effect of satellite accretion on the building-up of the radial stellar age distribution in the discs of spiral galaxies. In addition, we analyse its effect on other chemical and dynamical properties of these systems up to their outskirts.

With such aim, we made use of the simulated Milky Way mass, fully-cosmological discs from Ramses Disc Environment Study (RaDES, Few et al. 2012). We analysed their assembly histories by examining the proximity of satellites to the galactic disc to gauge which satellites impact the disc.

They can be divided into three stages affecting the stellar age-velocity dispersion (AVR) and age-metallicity relations (AMR): i) a merger dominated phase; ii) a quieter phase ending with a final merger event marking the onset of important radial differences in the AMR and AVR; and iii) a secular phase with few mergers not affecting the disc properties. Stars born during the first two phases display high velocity dispersions and a high degree of chemical mixing, in contrast to those born during phase iii (low velocity dispersion and low degree of chemical mixing). As a consequence of such assembly history we find:

- Inverted AMR trends in the outer parts of discs for phase iii stars.

- U-shaped stellar age profiles followed by an old plateau ( 10 Gyr) in the outer discs for the entire RaDES sample (regardless of their Surface Brightness profiles). This shape is recovered even in the absence of radial migration (the minimum in age is "older" in this case).

These recovered age profiles agree with a possible scenario in which star formation proceeds inside-out (inner negative gradient), there was an early build-up of the entire disc (outer old plateau), and radial motions smoothen the shape of the age profiles.

All these results have been already published in Ruiz-Lara et al. 2016. We strongly encourage the reader to check such paper to expand the information given in this proceeding and to check the whole extent of such work on the effect of the cosmological assembly history on the chemical and dynamical properties of the discs of spiral galaxies.

\section{References}

Few, C. G., Gibson, B. K., Courty, S., Michel-Dansac, L., Brook, C. B., \& Stinson, G. S. 2012, $A \mathscr{G} A, 547, \mathrm{~A} 63$

Ruiz-Lara, T., Few, C. G., Gibson, B. K., Pérez, I., Florido, E., Minchev, I., \& Sánchez-Blázquez, P. 2016, A\&A, 586, A112 